\title{
An evaluation of solid versus liquid transport media for high-risk HPV detection and cervical cancer screening on self- collected specimens
}

Hui Du ${ }^{1+}$, Xianzhi Duan ${ }^{2+}$, Yan Liu ${ }^{3+}$, Bin Shi ${ }^{4+}$, Wei Zhang ${ }^{5+}$, Chun Wang ${ }^{1}$, Xinfen Qu ${ }^{1}$, Juncui Bao ${ }^{1}$, Jingran Li ${ }^{6}$,

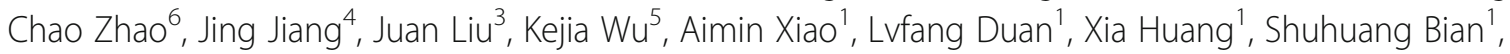

Lijie Zhang ${ }^{1}$, Hongxue Luo ${ }^{1}$, Lihui Wei ${ }^{6^{*}}$, Jerome L. Belinson ${ }^{7 *}$, Ruifang $\mathrm{Wu}^{{ }^{* *}}$ and CHIMUST group

\begin{abstract}
Background: The solid transport media is a small size card that allows fast, easy DNA extraction from a variety of biological samples. In 2016 we developed a solid media transport card; for that pilot study to control the selfcollection we used a pseudo-self-collection technique. The current study expands this prior work using true selfcollections and only the POI card, and aims to evaluate the solid media transport card to detect HR-HPV in selfsamples compared to liquid transport media.

Methods: Ten thousand eight hundred eighty-five women between the ages of 30-59 with no screening for 3 years were enrolled. The self-collected sample was first applied to a new solid media transport card (Labeled as SC) then the brush placed in $6 \mathrm{ml}$ ThinPrep liquid (Labeled as SL). Then a physician collected a direct endocervical specimen into ThinPrep liquid (Labeled as DL). Samples were tested with Cobas 4800 and the SeqHPV NGS assay for HR-HPV. Patients positive on any test were recalled for colposcopy and biopsy.

Results: Ten thousand three hundred thirty-nine participants had complete data. The mean age was 43.9 years. CIN $2+$ rates were 1.4\% (142/10339). The agreement in HPV detection between the two different self-sample collection media was also good (Cobas HPV kappa $=0.86$; SeqHPV kappa $=0.98$ ). Tested with Cobas, the sensitivity of Cobas-SL and Cobas-SC for CIN 2+ was95.07 and 94.37\%; and for CIN3+ was 96.30, 96.30\% respectively. The specificity of Cobas-SC, and Cobas-SL for CIN2+ was 88.74 and 87.35\%; for CIN3 was 88.04and 86.65\% respectively. Tested with SeqHPV, the sensitivity for CIN2+ of Seq-SC and Seq-SL was 95.77 and 96.48\%; for CIN3+, both the SC and SL specimens had a sensitivity of 100\%. The specificity for CIN2+ of Seq-SC and Seq-SL was 89.54 and 89.53\%; for CIN3+ was 88.84,88.82\% respectively. For both HR-HPV assays, the sensitivities were similar for the two self-sample media (SC vs SL, $p=1.00$ ).

(Continued on next page)
\end{abstract}

\footnotetext{
*Correspondence: weilhpku@163.com; jib@poiinc.org; wurfpush@126.com

${ }^{\dagger}$ Hui Du, Xianzhi Duan, Yan Liu, Bin Shi and Wei Zhang contributed equally to this work.

${ }^{6}$ Peking University People's Hospital, Beijing, PR China

${ }^{7}$ Preventive Oncology International Inc, and the Cleveland Clinic, Cleveland,

$\mathrm{OH}$, USA

'Peking University Shenzhen Hospital, Shenzhen, PR China

Full list of author information is available at the end of the article
}

C C The Author(s). 2020 Open Access This article is licensed under a Creative Commons Attribution 4.0 International License, which permits use, sharing, adaptation, distribution and reproduction in any medium or format, as long as you give appropriate credit to the original author(s) and the source, provide a link to the Creative Commons licence, and indicate if changes were made. The images or other third party material in this article are included in the article's Creative Commons licence, unless indicated otherwise in a credit line to the material. If material is not included in the article's Creative Commons licence and your intended use is not permitted by statutory regulation or exceeds the permitted use, you will need to obtain permission directly from the copyright holder. To view a copy of this licence, visit http://creativecommons.org/licenses/by/4.0/ The Creative Commons Public Domain Dedication waiver (http://creativecommons.org/publicdomain/zero/1.0/) applies to the data made available in this article, unless otherwise stated in a credit line to the data. 
(Continued from previous page)

Conclusions: The solid transport card for collecting vaginal self-samples as accurate as liquid transport media assayed by two different PCR based HR-HPV tests. The solid transport media is a suitable medium for collecting and storing vaginal self-samples.

Keywords: Cervical Cancer screening, High risk HPV testing, Solid media transport, Self-collection

\section{Introduction}

Traditionally cervical swabs have been placed in liquid media for transport. Due to the logistical difficulties such as spillage, flammability, and weight, adding to the risks and costs of liquid transport media, solid carriers consisting of chemically treated or untreated filter paper have been investigated for hrHPV testing. These filter paper cards are easy and safe to store and transport [1-13]. Recently we (Maurer, et.al.) designed and tested a new solid media transport card (POI card) that was compared to the established iFTA card from GE Healthcare. The new card performed equal to the iFTA card in terms of transfer of HPV DNA and sensitivity/specificity for CIN2+. In addition, the new card did not degrade in high humid environments like the iFTA card [14]. In a sub study (Luo et al.) provides data to suggest the iFTA may be a poorer transport vehicle than the new card when combined with the Cobas assay [15]. Therefore, in this trial we will evaluate the recently validated POI card for detecting HR-HPV in vaginal self-samples compared to self-collected samples transported in the standard PreservCyt liquid.

The Cobas 4800 HPV test, the first approved HPV assay for primary screening by US FDA, is a qualitative multiplex assay, providing specific genotyping information for HPV types 16 and 18, and then 12 other high-risk HPV types as a pooled result [16]. The SeqHPV test (BGI Shenzhen, Shenzhen, China) is a high throughput HPV genotyping assay based on multiplex PCR and next generation sequencing It is configured to detect 14 high risk types of HPV $(16,18,31,33,35,39,45,51,52,56,58,59,66$ and 68). These characteristics make it well suited for centralized laboratory processing in high volume screening programs. The assay has been previously validated using SHENCCAS T II specimens (Shenzhen Cervical Cancer Screening Trial [17]. Our study was designed to evaluate the performance of the Cobas 4800 HPV assay and SeqHPV for testing vaginal self-samples via either liquid or solid specimen transport method. Based on the multi-center population-based cervical cancer screening, we identified the concordance and the effectiveness in detecting high-risk HPV in different self-sample collection media.

\section{Materials and methods}

\section{Study population and design}

Between Aug 2016 to Aug 2018, a multi-center, population-based cross-sectional cervical cancer screening study [Chinese Multi-Center Screening Trial (CHIMUST)] was conducted in China.10,885 subjects were recruited from 15 screening sites in 7 provinces. Women in those sites were eligible if they were 25-59 years of age, sex-experienced but not pregnant, had no cervical cancer screening for at least 3 years, no prior hysterectomy, and no prior pelvic radiation. The study protocol was approved by the human subject review boards of the Peking University Shenzhen Hospital (PUSH), Shenzhen, China, and the Cleveland Clinic, Cleveland, USA. In addition, the study was registered with the Chinese Clinic Trial Registry (chiCTR-EOC16008456), an international clinical trials registry platform approved by the WHO. Women with incomplete data were excluded from the analyses. This included: (1) women testing positive by any HPV test without having colposcopy and biopsies; (2) Specimen is not sufficient or no sample for an HPV test; (3) Failure of any HPV test.

\section{Study samples collection}

Every woman had contributed two specimens, one was collected by herself (Self-Sample), and one was collected from the endocervix by a clinician (Direct-Sample). The self-collected sample was first applied to the solid media transport card (labeled as SC-sample), then the brush placed in $6 \mathrm{ml}$ ThinPrep ${ }^{\oplus}$ PreservCyt $^{\circ}$ Solution (Hologic Bedford, MA, USA) (labeled as SL-sample). The brush was then agitated in $6 \mathrm{ml}$ PreservCyt Liquid (as a split sample). Self-sampling instructions were provided by poster diagrams and personal instruction. The physician-collected samples were placed in $20 \mathrm{ml}$ ThinPrep ${ }^{\curvearrowleft}$ PreservCyt ${ }^{\oplus}$ Solution (labeled as DL-sample). All samples were stored at room temperature and were tested with Cobas $4800 \mathrm{HPV}$ test and SeqHPV test for HR-HPV within 2 months of collection. Patients testing HPV positive (self or direct), were recalled for colposcopy with directed and random 4 quadrants microbiopsies plus endocervical curettage (ECC). Histology slides were interpreted by a gynecologic pathologist from PUSH (Author C.W). Immunochemical staining with p16 was selectively obtained to adjudicate difficult cases.

\section{The solid transport card management protocol}

The new solid transport card consists of PK $226^{\circ}$ paper (PerkinElmer, Greenville, SC) treated with a 
Table 1 The prevalence of HPV

\begin{tabular}{llll}
\hline HPV Screening tests & The prevalence of HPV & HPV Screening tests & The prevalence of HPV \\
\hline Cobas-DL(doctor liquid) & $10.8 \%$ & Seq--DL(doctor liquid) & $10.9 \%$ \\
Cobas-SL (self liquid) & $13.7 \%$ & Seq--SL(self liquid) & $11.6 \%$ \\
Cobas-SC(self card) & $12.4 \%$ & Seq-SC(self card) & $11.6 \%$
\end{tabular}

Note: $D L$ Doctor liquid, endocervical samples in liquid media; SL Self liquid, cervicovaginal specimen in liquid media; SC Self card, cervicovaginal specimen in solid transport card

combination of a lysing solution and a dye (Hyde Biomedical Corporation, Wuhu, Anhui, China). The lysing solution contains an ionic detergent to lyse cell membranes and stabilize DNA as well. Similar, to the FTA card, the indicating dye changes color when the sample is applied. The sample area on the card was punched using a 5-mm Harris micro-punch (BSD, USA). Each card was manually punched 3 times and placed in a single well in a 96-well plate. Then they were all washed once using $100 \mu \mathrm{l}$ of sterile water. The water is carefully removed with a sterile fine-tip pipette. The DNA elution is performed with $50 \mu \mathrm{l}$ of sterile water at $56^{\circ} \mathrm{C}$ for 30 min immediately followed by $95^{\circ} \mathrm{C}$ for $15 \mathrm{~min}$. in a heating block. The 96-well plate containing DNA elution and pieces of card are then centrifuged at $4000 \mathrm{rpm}$ for $3 \mathrm{~min}$ and the eluted DNA is transferred into a new 96well plate. When performing the assays, $5 \mathrm{ul}$ of DNA will be used in each well of the 96 well plates for PCR, for Cobas assay, and SeqHPV. Our prior study shows that $5 \mathrm{ul}$ is the standard volume used for the Cobas assay, and SeqHPV, and it has been thoroughly tested and demonstrated to be optimal with $>99 \%$ adequate specimens. (Any storage for future use will be at $-80^{\circ} \mathrm{C}$ ) $[14,15]$.

\section{High risk HPV detection}

All samples were tested with Cobas 4800 HPV test and SeqHPV test for HR-HPV according to the manufacturer's instructions. All the DL-Samples and SL-samples accepted by the PUSH lab were split, after thoroughly mixed by shaking, $1 \mathrm{cc}$ used for Cobas 4800 and $1 \mathrm{cc}$ for SeqHPV testing. After splitting, the physician-collected samples were processed for cytology interpretation. The Cobas 4800 system platform (Roche Molecular Diagnostics, Pleasanton, CA.), consists of the Cobas $\times 480$ instrument and the Cobas z480 analyzer. We used two different specimen preparation procedures for the Cobas z480 that had previously been optimized. Nucleic acids used for the Cobas assay from the two liquid specimens $(\mathrm{DL}$ and SL), were prepared using Cobas $\times 480$. The instrument could yield $50 \mu \mathrm{L}$ of nucleic acid, eluted from $500 \mu \mathrm{L}$ of ThinPrep solution used per subject. The eluted DNA solutions from the solid cards were prepared according to the Cobas4800 device "special instructions". The "PCRONLY" program was performed using Cobas z480.5 $\mu \mathrm{L}$ DNA solution from the solid card samples were needed to ensure a sufficient sample (adequate DNA) was present for valid detection.

The "split sample" methodology we used, was used extensively in the 1990's for the development of liquid based cytology [18]. The Both of HPV assays we studied were designed using a human $\beta$-globin gene $(\mathrm{HBB}$, House keeping gene) as an internal control to identify false negatives caused by inadequate DNA or failed PCR.

\section{Cytology}

Cytology using the Hologic I2 imager systems (computer assisted diagnosis) will be used for future research not for patient care in the current study.

\section{Statistical analysis}

Data were entered in an ACCESS database specially designed for CHIMUST. To evaluate the effectiveness of different sample collection media for vaginal selfsamples in population-based screening, we calculated sensitivities for detecting $\mathrm{CIN} 2+$ and $\mathrm{CIN} 3+$, the concordance in detecting high-risk HPV, and the differences in HPV assay performance using solid (filter paper cards) and liquid based specimen transport for the selfcollected and physician collected cervico-vaginal specimens. Sensitivity and specificity of the Cobas and SeqHPV testing results in detecting high-risk HPV and $\mathrm{CIN} 2+$ were calculated using $\mathrm{CIN} 2+$ as the endpoint. McNemar's Chi-square was performed to calculate differences between paired proportions at a probability level of 0.05. Agreement between self and direct samples was measured by absolute agreement and Kappa statistics (Cohen's Kappa). Agreement between the solid transport media and liquid transport media for detecting

Table 2 The concordance in detecting high-risk HPV between Cobas SL and SC

\begin{tabular}{|c|c|c|c|c|c|c|c|}
\hline & & \multicolumn{2}{|c|}{ Cobas-SL } & \multirow{2}{*}{$\begin{array}{l}\text { \% Positive } \\
\text { Agreement }\end{array}$} & \multirow{2}{*}{$\begin{array}{l}\% \\
\text { Negative } \\
\text { Agreement }\end{array}$} & \multirow{2}{*}{$\begin{array}{l}\% \text { Overall } \\
\text { Agreement }\end{array}$} & \multirow{2}{*}{$\begin{array}{l}\text { Kappa Value } \\
{[95 \% \mathrm{Cl}]}\end{array}$} \\
\hline & & + & - & & & & \\
\hline \multirow[t]{2}{*}{ Cobas-SC } & + & 1197 & 236 & 92.21 & 97.40 & 96.84 & $0.86[0.85-0.88]$ \\
\hline & - & 92 & 8874 & & & & \\
\hline
\end{tabular}


Table 3 The concordance in detecting high-risk HPV between Seq-SL and Seq-SC

\begin{tabular}{|c|c|c|c|c|c|c|c|}
\hline & & Seq-S & & \% Positive & & $\%$ Overall & Kappa Value \\
\hline & & + & - & & $\begin{array}{l}\text { Negative } \\
\text { Agreement }\end{array}$ & Agreement & \\
\hline Seq-SC & + & 1185 & 24 & 97.85 & 99.73 & 99.52 & $0.98[0.97-0.98$ \\
\hline & - & 26 & 9164 & & & & \\
\hline
\end{tabular}

HR-HPV in vaginal self-samples was also measured. All data were analyzed using SPSS 17.0.

\section{Results}

\section{Demographic characteristics of participants}

Ten thousand three hundred ninety-nine women had complete data. Mean age was 43.9 years. The return rate for colposcopy was $81.0 \%$. 1.4\% (141 patients) had CIN2+ and $0.5 \%$ (or 54 patients) had CIN $3+.101$ $(0.93 \%)$ women were dropped from the analysis due to HPV test failure. 6 (0.05\%), 2 (0.02\%), 29 (0.27\%),34 (0.31\%), 19 (0.17), and 19 (0.17) were missing Cobas-DL, Cobas-SL, Cobas-SC, Seq HPV-DL, SeqHPV-SL and SeqHPV-SC, respectively. Testing failure in both the assays were reported by the lab as inadequate DNA (Table supplement 1).

\section{HPV positivity in different collection media}

Overall the HPV infection rates were $10.8 \%$ for Cobas and $10.9 \%$ for SeqHPV in clinician-collected samples (labeled as DL-sample), and $13.7 \%$ for Cobas and $11.6 \%$ for SeqHPV in self-samples placed in liquid medium (labeled as SL-sample), and $12.4 \%$ for Cobas and $11.6 \%$ for SeqHPV in self-samples collected on the solid transport card (labeled as SC-sample) (Table 1).

\section{HPV concordance for the two different self-sample collection media}

Tables 2 and 3 show the agreement in HPV detection between the two different self-sample collection media (Solid media vs liquid media) was very good (Cobas HPV kappa $=0.86$; SeqHPV kappa $=0.98$ ).

\section{Accuracy of detecting CIN2+, CIN3+ in different self- sample collection media}

Tables 4 and 5 show the sensitivity and specificity of different self-sample collection media for $\mathrm{CIN} 2+$ and
CIN3+ along with confidence intervals and $p$-values. Tested with Cobas, the sensitivity of Cobas-SC and Cobas-SL for CIN $2+$ was 94.37 and $95.07 \%$; and for CIN3+ was 96.30 and $96.30 \%$ respectively. The specificity of Cobas-SC, and Cobas-SL for CIN2+ was 87.74 and $88.35 \%$; and for CIN3 was 88.04 and $86.65 \%$ respectively. Tested with SeqHPV, the sensitivity for CIN2+ of Seq-SC and Seq-SL was 95.77and 96.48\%; and for CIN3+, both the SC and SL specimens had a sensitivity of $100 \%$. The specificity for CIN2+ of Seq-SL and Seq-SC was 89.54 and $89.53 \%$; and for CIN3+ was 88.84 and $88.82 \%$ respectively. For the two HPV assays, there was no significant difference in sensitivity for both the detection of CIN2+ and CIN3+ between SC and SL $(p=1.00)$. The card (SC) was significantly more specific than the selfliquid sample (SL) on the Cobas assay but similar with SeqHPV for both CIN2+ and CIN3+ (Tables 4 and 5).

\section{Discussion}

As noted in the introduction, the current study expands our prior work using "true" self-collections and only the POI card. We still used the split sample method to generate the SC (card) and the SL (liquid) samples. Our data shows that the POI Card performed well in this selfcollection trial demonstrating equal sensitivity to liquid samples run both on Cobas and SeqHPV. We know from our prior work in SPOCCS III, that self-collected specimens will identify more HPV than direct endocervical specimens. This excess HPV found in the vagina is unassociated with CIN in the cervix [19]. Of interest it appears that more cellular DNA was washed from the brush in the secondary liquid split self-samples. This appears to have not affected the SeqHPV assay due to its analytical sensitivity but did the Cobas assay. In addition, even with a non-significant increase in sensitivity, since the SeqHPV assay has variable cut-points by HPV type

Table 4 The sensitivity and specificity for $\geq$ CIN 2 for the Cobas and SeqHPV assays

\begin{tabular}{|c|c|c|c|c|c|}
\hline HPV tests & Sensitivity $(95 \% \mathrm{Cl})$ & $P$ value & Specificity $(95 \% \mathrm{Cl})$ & $P$ value & NPV ((95\% Cl) \\
\hline Cobas--SL & $95.07(89.72,97.82)$ & - & $87.35(86.68,87.98)$ & - & $99.92(99.83,99.97)$ \\
\hline Cobas-SC & $94.37(88.83,97.36)$ & 1.0 & $88.74(88.11,89.34)$ & $<0.001$ & $99.91(99.82,99.96)$ \\
\hline Seq--SL & $96.48(91.55,98.70)$ & - & $89.53(88.92,90.11)$ & - & $99.95(99.87,99.98)$ \\
\hline Seq-SC & $95.77(90.63,98.27)$ & 1.0 & $89.54(88.93,90.12)$ & 0.89 & $99.93(99.85,99.97)$ \\
\hline
\end{tabular}

Note: $\geq$ CIN2 Cervical intraepithelial neoplasia 2 or higher, $95 \% \mathrm{Cl} 95 \%$ confidence interval

The comparison of solid vs. liquid transport media for detecting HR-HPV in vaginal self-collected specimens were calculated using the McNemar's Chi-square test 
Table $\mathbf{5}$ The sensitivity and specificity for $\geq$ CIN $\mathbf{3}$ for the Cobas and SeqHPV assays

\begin{tabular}{llllll}
\hline HPV tests & Sensitivity $\mathbf{( 9 5 \% C l )}$ & $\boldsymbol{P}$ value & Specificity $\mathbf{( 9 5 \% ~ C l )}$ & $\boldsymbol{P}$ value & NPV $(\mathbf{9 5} \% \mathbf{C l})$ \\
\hline Cobas--SL & $96.30(86.16,99.36)$ & - & $86.65(85.98,87.30)$ & - & $99.98(99.91,100)$ \\
Cobas -SC & $96.30(86.16,99.36)$ & 1.0 & $88.04(87.40,88.66)$ & $<0.001$ & $99.98(99.91,100)$ \\
SEQ --SL & $100(91.73,100)$ & - & $88.82(88.10,89.41)$ & - & $100(99.95,100)$ \\
SEQ -SC & $100(91.72,100)$ & 1.0 & $88.84(88.21,89.43)$ & 0.78 & $100(99.95,100)$ \\
\hline
\end{tabular}

Note: $\geq$ CIN3 Cervical intraepithelial neoplasia 3 or higher

based on the likelihood of causing high grade CIN, it did not affect specificity.

In 2016 we published our work developing a solid media transport card [14]. The new card project was initiated for several reasons: First we anticipated the need to collect thousands of samples per day and realized that the common alcohol (methanol, ethanol) containing liquid transport medias were a risk for children and spillage when home collections were involved. Second, the daily transport of these alcohol containing liquid specimens was complex and expensive. Third, a solid media transport seemed to be a good alternative but the commercial cards available at the time (iFTA, G.E. Healthcare) were expensive, especially for our medically underserved populations. In addition, in our initial trials the iFTA deteriorated in high humid environments.

After developing the POI card, we published a second study comparing liquid (SurePath) vs solid media (iFTA and POI cards) with Cobas. For this pilot study to control the self-collection we used a pseudo-self-collection technique (physician collects cervico-vaginal sample without using a vaginal speculum) [15]. We were both surprised and puzzled to discover the Cobas4800 test in combination with iFTA card (FTA) was inferior to the other samples studied (liquid self, POI card, direct liquid), both for the detection of HrHPV as well as CIN2/ $3+$. This was especially surprising for the comparison "liquid self" to "FTA", since the liquid self-sample was a secondary sample (split-sample) from FTA (the primary sample).

In a study from Sweden evaluating the HRVIR assay (laboratory developed test) compared to Cobas using FTA cards, the clinical sensitivity of the FTA card and HPVIR test was equivalent to Cobas, and the Cobas assay detected 63 of 67 women with CIN2+ [94.0\% (95\% $\mathrm{CI}=85.2-98.1)] \quad[20]$. Likewise, Dong et.al. evaluated FTA and liquid samples with Cobas in a small study and also found good concordance. Interestingly, they found the same concordance for dry brush samples which for cost and simplicity is an important future direction [21].

In our trial when split samples were used, we have twice encountered discordant results, once with the primary card sample and once with the secondary liquid sample. Not with standing, we believe the strength of our work and the current literature supports the equivalency of solid media specimen transport, and liquid transport methodologies. The potential impact for non-liquid specimen transport on mass populationbased screening is profound. Especially in current times of COVID, where self-collection methodologies will be preferable than bringing large populations together for move conventional cervical cancer screening events.

\section{Conclusions}

We believe that the current study adds considerable power to the literature on specimen media comparisons, with 141 cases of CIN2+. The use of non-liquid forms of specimen transport, especially integrated with selfcollection sampling screening programs appears to demonstrate equivalency and is an important area for future study and implementation.

\section{Supplementary Information}

The online version contains supplementary material available at https://doi. org/10.1186/s13027-020-00333-4.

Additional file 1: Table supplement 1. Rate of HPV testing failure.

\section{Acknowledgements}

We appreciate gynecologists and pathologists in our hospital for assisting in preparation of this manuscript, and thank all the women participated in this study.

\section{Authors' contributions}

Conceptualization: Ruifang Wu, Jerome L. Belinson, Lihui Wei, Hui Du. Methodology: Ruifang Wu, Jerome L. Belinson, Hui Du. Software: Xinfen Qu, Hui Du. Formal analysis: Hui Du, Ruifang Wu, Jerome L. Belinson. Investigation: Hui Du, Xianzi Duan, Yan liu, Bin Shi, Wei Zhang, Xia Huang Resources: Ruifang Wu, Hui Du. Data curation: Hui Du, Ruifang Wu. Writing original draft: Hui Du, Jerome L. Belinson. Writing - review \& editing: Ruifang Wu, Jerome L. Belinson. Supervision: Ruifang Wu, Hui Du. Project administration: Ruifang Wu, Hui Du, Jerome L. Belinson, Lihui Wei, Xianzi Duan, Yan liu, Bin Shi, Wei Zhang, Chun Wang, Xinfen Qu, Jingran Li, Chao Zhao, Jing Jiang, Juan Liu, Kejia Wu, Aimin Xiao, Juncui Bao, Lvfang Duan, Xia Huang, Hongxue Luo, Shuhuang Bian, Lijie Zhang. Funding acquisition: Ruifang Wu, Hui Du. The author(s) read and approved the final manuscript.

\section{Funding}

This work was supported by the China International Exchange and Promotion Association for medical and Healthcare; Shenzhen Sanming Project (SZSM2020); Shenzhen High-level Hospital Construction Fund; Shenzhen Key Medical Discipline Construction Fund (No.SZXK027); the Shenzhen Health Family Planning Commission under the Foundation Clinical Research Projects (SZLY2017005); the Peking University Shenzhen Hospital. 


\section{Availability of data and materials}

All data generated or analyzed during this study are included in this article. The datasets used and/or analyzed during the current study are available from the corresponding author on reasonable request.

\section{Ethics approval and consent to participate}

All procedures in studies involving human participants were performed in accordance with ethical standards and approved by Both the Peking University Shenzhen Hospital (Shenzhen, China) and the Cleveland Clinic (Cleveland, Ohio) an Institutional Review Boards.

\section{Consent for publication}

The manuscript was approved for publication by all authors.

\section{Competing interests}

The funders had no role in study design, in the collection, analysis and interpretation of data, and decision to publish, or preparation of the manuscript. All the authors declare no conflicts of interests.

\section{Author details}

${ }^{1}$ Peking University Shenzhen Hospital, Shenzhen, PR China. ${ }^{2}$ Capital Medical University Beijing Tongren Hospital, Beijing, PR China. ${ }^{3}$ Fudan University Huashan Hospital North, Shanghai, PR China. ${ }^{4}$ The second Hospital of Hebe Medical University, Shijiazhuang, PR China. ${ }^{5}$ Wuhan University Zhongnan Hospital, Wuhan, PR China. ${ }^{6}$ Peking University People's Hospital, Beijing, PR China. ${ }^{7}$ Preventive Oncology International Inc, and the Cleveland Clinic, Cleveland, OH, USA.

Received: 11 June 2020 Accepted: 28 October 2020

Published online: 30 November 2020

\section{References}

1. Pitcovski J, Shmueli E, Krispel S, Levi N. Storage of viruses on filter paper for genetic analysis. JVirol Methods. 1999;83:21-6.

2. Kailash U, Hedau S, Gopalkrishna V, Katiyar S, Das BC. A simple 'paper smear' method for dry collection, transport and storage of cervical cytological specimens for rapid screening of HPV infection by PCR. J Med Microbiol. 2002:51:606-10.

3. Gustavsson I, Lindell MF, Wilander EF, Strand AF, Gyllensten U. Use of IFTA card for dry collection, transportation and storage of cervical cell specimen to detect high-risk HPV. J Clin Virol. 2009;46:112-6.

4. Gustavsson I, Sanner K, Lindell M, Strand A, Olovsson M, Wikström I, Wilander E, Gyllensten U. Type-specifific detection of high-risk human papillomavirus (HPV) in self-sampled cervicovaginal cells applied to iFTA elute cartridge. J Clin Virol. 2011;51:255-8.

5. Lenselink CH, DeBie RP, van Hamont D, Bakkers JM, Quint WG, Massuger LF, Bekkers RL, Melchers WJ. Detection and genotyping of human papillomavirus in self-obtained cervicovaginal samples by using the FTA cartridge: new possibilities for cervical cancer screening. J ClinMicrobiol. 2009;47:2564-70.

6. DeBie RP, Schmeink CE, Bakker JM, Snijder PJ, Quint WG, Massuger LF, Bekkers RL, Melchers WJ. The indicating FTA elute cartridge a solid sample carrier to detect high-risk HPV and high-grade cervical lesions. J Mol Diagn. 2011;13:371-6

7. Gonzalez P, Cortes B, Quint W, Kreimer AR, Porras C, Rodriguez AC, Jimenez S, Herrew R, Struijk L, Hildesheim A, Melchers W. Evaluation of the IFTA carrier device for human papillomavirus testing in developing countries. J Clin Microbiol. 2012;50:3870-6.

8. Geraets DT, van Baars R, Alonso I, Ordi J, Torné A, Melchers WJ, Meijer CJ, Quint WG. Clinical evaluation of high-risk HPV detection on self-samples using the indicating FTA-elute solid-carrier cartridge. J Clin Virol. 2013;57: 125-9.

9. Guan Y, Gravitt PE, Howard R, Eby YJ, Wang S, Li B, Feng C, Qiao YL, Castle PE. Agreement for HPV genotyping detection between self-collected specimens on aiFTA cartridge and clinician-collected specimens. J Virol Methods. 2013;189:167-71.

10. Phongsavan K, Gustavsson I, Marions L, Phengsavanh A, Wahlstrom R, Gyllensten U. Detection of human papillomavirus among women in Laos: feasibility of using fifilter paper card and prevalence of high-risk types. Int J Gynecol Cancer. 2012;22:1398-406.
11. Ayele W, Schuurman R, Messele T, Dorigo-Zetsma W, Mengistu Y, Goudsmit J, Paxton WA, de Baar MP, Pollakis G. Use of dried spots of whole blood, plasma, and mother's milk collected on fifilter paper for measurement of human immunodefificiency virus type 1 burden. J Clin Microbiol. 2007;45: 891-6.

12. Santos CR, Franciscatto LG, Barcellos RB, Almeida SE, Rossetti ML. Use of FTA elute card impregnated with cervicovaginal sample directly into the amplifification reaction increases the detection of human papillomavirus DNA. Braz J Microbiol. 2012;43:389-92.

13. Smit PW, Elliott I, Peeling RW, Mabey D, Newton PN. An overview of the clinical use of fifilter paper in the diagnosis of tropical diseases. Am J Trop Med Hyg. 2014;90(2):195-210.

14. Maurer K, Luo H, Shen Z, Wang G, Du H, Qu X, Wu R, Belinson JL. The Development and Evaluation of a New Solid Media Specimen Transport Card for Population Based Cervical Cancer Prevention. J Clin Virol. 2016;76: $14-9$.

15. Luo H, Du H, Maurer K, Belinson JL, Wang G, Liu Z, Zhang L, Zhou Y, Wang C, Tang J, Qu X, Wu R. An evaluation of the Cobas4800 HPV test on Cervicovaginal specimens in liquid versus solid transport media. PLoS One. 2016;11: e0148168.

16. Castle PE, Stoler MH, Wright TC Jr, Sharma A, Wright TL, Behrens CM. Performance of carcinogenic human papillomavirus (HPV) testing and HPV16 or HPV18 genotyping for cervical cancer screening of women aged 25 years and older: a subanalysis of the ATHENA study. Lancet Oncol. 2011; 12:880-90.

17. Yi X, Zou J, Xu J, Liu T, Liu T, Hua S, Xi F, Nie X, Ye L, Luo Y, et al. Development and validation of a new HPV genotyping assay based on next generation sequencing. Am J Clin Pathol. 2014;141:796-804.

18. Lee KR, Ashfaq R, Birdsong GG, Korkill ME, Mcintosh KM, Inhorn SL. Comparison of conventional Papanicolaou smears and a fluid-based thinlayer system for cervical cancer screening. Obstet Gynecol. 1997;90:278-84.

19. Belinson JL, Hu S, Niyazi M, Pretorius RG, Wang H, Wen C, Smith J, Li J, Taddeo F, Burchette R, Qiao YL. Prevalence of type-specific human papillomavirus in endocervical, upper and lower vaginal, perineal, and vaginal self-collected specimens; implications for vaginal self-collection. Int J Gyn Cancer. 2010;127(5):1151-7.

20. Gustavsson I, Aarnio R, Myrnäs M, Hedlund-Lindberg J, Taku O, Meiring T, Wikström I, Enroth S, Williamson AL, Olovsson M, Gyllensten U. Clinical validation of the HPVIR high-risk HPV test on cervical samples according to the international guidelines for human papillomavirus DNA test requirements for cervical cancer screening. Virol J. 2019;16:107.

21. Dong L, Lin C, Li L, Wang M, Cui J, Feng R, Liu B, Wu Z, Lian J, Liao G, et al. An evaluation of clinical performance of FTA cards for HPV 16/18 detection using cobas $4800 \mathrm{HPV}$ test compared to dry swab and liquid medium. J Clin Virol. 2017;94:67-71.

\section{Publisher's Note}

Springer Nature remains neutral with regard to jurisdictional claims in published maps and institutional affiliations.
Ready to submit your research? Choose BMC and benefit from:
- fast, convenient online submission
- thorough peer review by experienced researchers in your field
- rapid publication on acceptance
- support for research data, including large and complex data types
- gold Open Access which fosters wider collaboration and increased citations
- maximum visibility for your research: over $100 \mathrm{M}$ website views per year
At BMC, research is always in progress.
Learn more biomedcentral.com/submissions 